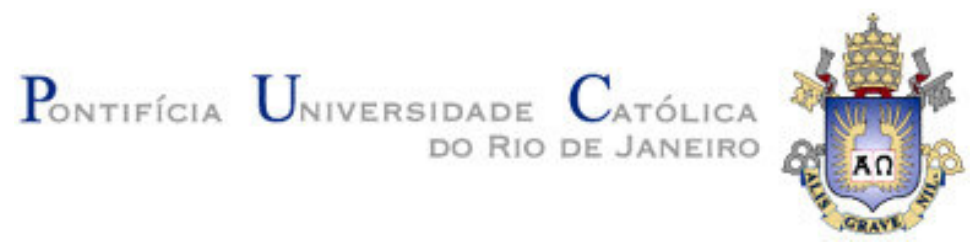

Eduardo Henrique de Sousa Salvino

Sistema de doações pela internet para a ajuda humanitária

Dissertação apresentada como requisito parcial para obtenção do título de Mestre pelo Programa de PósGraduação em Engenharia de Produção do Departamento de Engenharia Industrial da PUC-Rio.

Orientador: Prof. Dr. José Eugenio Leal

Rio de Janeiro

Setembro de 2012 


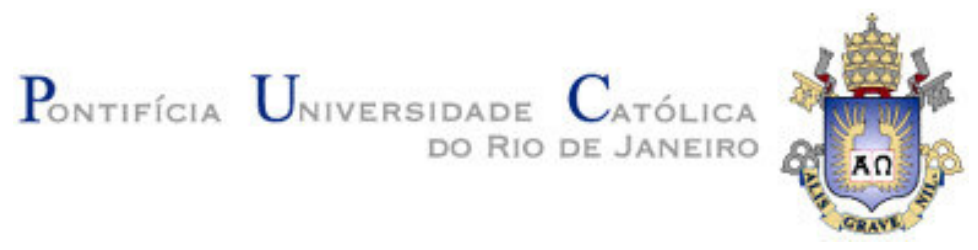

Eduardo Henrique de Sousa Salvino

\section{Sistema de doações pela internet para a ajuda humanitária}

Dissertação apresentada como requisito parcial para obtenção do título de Mestre pelo Programa de PósGraduação em Engenharia de Produção do Departamento de Engenharia Industrial da PUC-Rio. Aprovada pela Comissão Examinadora abaixo assinada.

Prof. Dr. José Eugênio Leal

Orientador

Departamento de Engenharia Industrial - PUC-Rio

Prof. Dr. Luis Felipe Scavarda

Departamento de Engenharia Industrial - PUC-Rio

Prof. Dr. José Roberto Blaschek

Pontifícia Universidade Católica do Rio de Janeiro - PUC-Rio

Prof. Dr. José Eugênio Leal Coordenador Setorial do Centro Técnico Científico - PUC-Rio

Rio de Janeiro, 26 de setembro de 2012. 
Todos os direitos reservados. É proibida a reprodução total ou parcial do trabalho sem autorização da universidade, do autor e do orientador.

\section{Eduardo Henrique de Sousa Salvino}

Graduou-se em Engenharia Elétrica com ênfase em Telecomunicações e Sistemas de apoio à decisão na PUC-Rio em 2000. Especializou-se em Rede de Computadores na PUC-Rio em 2005. Atualmente trabalha com Suporte e Desenvolvimento no Departamento de Engenharia Industrial da PUC-rio.

Ficha Catalográfica

Salvino, Eduardo Henrique de Sousa

Sistema de doações pela internet para a ajuda humanitária / Eduardo Henrique de Sousa Salvino; orientador: José Eugenio Leal. - 2012.

72 f. : il. (color.) ; $30 \mathrm{~cm}$

Dissertação (mestrado)-Pontifícia Universidade

Católica do Rio de Janeiro, Departamento de Engenharia Industrial, 2012.

Inclui bibliografia

1. Engenharia Industrial - Teses. 2. Logística. 3. Logística humanitária. 4. Sistema de donativos. 5. Assistência humanitária. I. Leal, José Eugênio. II. Pontifícia Universidade Católica do Rio de Janeiro. Departamento de Engenharia Industrial. III. Título.

CDD: 658.5 
Os sonhos de Deus são maiores e melhores que os meus. 


\section{Agradecimentos}

Ao autor e consumador de minha fé, Senhor Jesus Cristo, por toda força e confiança em mim depositada. Nos momentos mais difíceis em que a conclusão parecia impossível, Ele com sua forte mão me encorajou e me conduziu ao termino de desta obra, que é para Sua honra e glória.

A Minha amada esposa Uleide Lima da Silva Salvino e a minha filha Joana Lima da Silva Salvino, pelo carinho e amor que me dedicaram, e pelo sacrifício dos muitos de seus fins de semana, os quais me fizeram companhia para que pudesse desenvolver este trabalho. Amo vocês.

Aos meus pais Reinaldo Henrique Salvino e Maria Trindade de Sousa Salvino, que sempre estiveram presentes nas inúmeras etapas de minha vida. O meu irmão Leonardo Henrique de Sousa Salvino, que me ajudou a montar a estrutura da base de dados, o modelo de dados e pela amizade de sempre. A minha irmã Janaína de Sousa Salvino, pela alegria de sempre em nossas vidas.

Ao meu orientador e amigo Prof. José Eugenio Leal, que com muita paciência me ajudou a elaboração e condução correta deste trabalho. A minha coordenadora Prof. ${ }^{a}$ Fernanda Maria Pereira Raupp, pela compreensão e colaboração para a montagem e revisão deste trabalho. Ao Prof. Madiagne Diallo pelo incentivo e apoio para que eu ingressasse no Programa de Mestrado Acadêmico. Ao Prof Tara Keshar Nanda Baidya, pela auxílio e orientação concedidos ao longo do curso e pela confiança .

A Vice Reitoria Acadêmica e a PUC-Rio, pela bolsa e por me liberar das minhas funções de funcionário para assistir as aulas do curso.

Aos meus amigos Funcionários Fernanda Lopes de Frias, Isabel Regina Finoti Loureiro, Celi Bernardo, Ana Zélia Martins, Gilvan Souza Nascimento e em especial Claudia Guimaraes Teti, pela intercessão junto a PUC-Rio para curso de mestrado.

A todos os professores do Departamento de Engenharia Industrial, pelo apoio e encorajamento em todos os momentos.

Aos amigos Antonio Ricardo Mesquita Jr, Denilson Ricardo de Lucena Nunes; Tatiana Fontes Cunha, Augusto da Cunha Reis e David Leite Carrilho pelas palavras de encorajamento e por todas as vezes que estudamos juntos para os diversos exames ao longo do curso. A nutricionista, Dr $^{\mathrm{a}}$ Claudia Cravo, pelas informações relativas à alimentação, as quais foram essenciais para dar maior consistência ao projeto de pesquisa. 
A amiga Rose Marinho, que também colaborou na revisão deste trabalho, lendo e discutindo os pontos em que não ficaram claros.

Ao amigo e irmão em Cristo Thiago Oliveira Santos, pela intercessão e apoio espiritual nas horas mais difíceis, meu agradecimento por estarmos sempre juntos. 


\section{Resumo}

Salvino, Eduardo Henrique de Sousa; Leal, José Eugênio. Sistema de doações pela internet para a ajuda humanitária. Rio de Janeiro, 2012. 72p. Dissertação de Mestrado - Departamento de Engenharia Industrial, Pontifícia Universidade Católica do Rio de Janeiro.

Diversas atividades humanas e constantes mudanças climáticas têm contribuído para o acontecimento de desastres em várias regiões do globo terrestre, intensificando a vulnerabilidade natural e socioeconômica nessas regiões. Estudiosos, agências governamentais e instituições não governamentais têm concentrado esforços para criar novos procedimentos de atenuação dos desastres e a favor de assistência humanitária. A necessidade de prestar assistência humanitária eficazmente às vítimas de desastre no menor tempo possível fez surgir uma nova área do conhecimento, a logística humanitária. Aliando conhecimentos de logística e de gestão da cadeia de suprimentos empresarial, a logística humanitária vem aplicar teoria e práticas correspondentes em prol das pessoas afetadas pelos eventos de desastre. Esta dissertação de mestrado tem como objetivo contribuir para a melhora do procedimento de arrecadação de doações, evitando o desvio e agilizando o processo de compra e entrega de materiais à população necessitada. Para isso, foi criado um sistema computacional que, através de uma página na internet, recebe doações e as redireciona para uma conta bancária a ser determinada, tendo como interface uma loja virtual que mostrará primeiramente a necessidade de suprimentos na região do desastre (alimentos, medicamentos, vestuário, entre outros) e depois o valor monetário total dos itens e quantidades escolhidas pelo doador, que poderá ser pago pelos principais meios disponíveis (cartão de credito, débito, transferência, boleto). Em particular, o sistema proposto leva em conta o deslocamento dos materiais, os depósitos de materiais existentes e o preço dos materiais nos fornecedores para obter o menor custo.

\section{Palavras-chave}

Logística; Logística Humanitária; Sistema de Donativos; Assistência Humanitária. 


\section{Abstract}

Salvino, Eduardo Henrique de Sousa; Leal, José Eugênio (Advisor). Donation System by Internet for humanitarian relief. Rio de Janeiro, 2012. 72p. Dissertação de Mestrado - Departamento de Engenharia Industrial, Pontifícia Universidade Católica do Rio de Janeiro.

Various human activities and constant changing in the climate have contributed to the occurrence of disasters in various regions of the globe, enhancing the natural and socio-economic vulnerability in these regions. Scholars, government agencies and non-governmental institutions have concentrated efforts to create new procedures to mitigate disasters and promote humanitarian assistance. The need to effectively provide humanitarian relief to victims of disaster in the shortest possible time introduced a new area of knowledge, the humanitarian logistics. Combining knowledge of logistics and supply chain management business, humanitarian logistics is to apply those theory and corresponding practices in support of people affected by disaster events. This dissertation aims to contribute to the improvement of the procedure of collecting donations, avoiding the detour and streamlining the purchasing process and delivery of materials to the needy population. For this, we created a computer system that, through a website, receives donations and redirects them to a bank account to be determined, with the interface of a virtual store that will show first the supply needs in the disaster area (food, medicine, clothing, etc.) and then the total monetary value of the items and quantities chosen by the donor, which may be paid by the principal means available (credit card, debit card, transfer, billet). In particular, the proposed system takes into account the displacement of material, the existing deposits of materials and the price of the materials from suppliers to obtain the lowest total cost, that is, the total amount donated corresponding to the lowest prices of materials and the smallest displacements to the location of disaster, considering the capacity the deposits.

\section{Keywords}

Logistics; Humanitarian Logistics; Donation Systems; Humanitarian Relief 


\section{Sumário}

1 INTRODUÇÃO 13

2 REVISÃO BIBLIOGRÁFICA 16

2.1. LOGÍSTICA 16

2.2. Desastres e Resposta a Desastres 18

2.3. LOCALIZAÇÃO DE FACILIDADES EM MEIO À EMERGÊNCIAS 23

2.4. LOGÍSTICA HUMANITÁRIA 23

2.5. BUSCA POR FORNECEDORES LOCAIS 27

2.6. Rota de Entrega dos ReCursos HumanitÁRIOS 27

3 CADEIA DE SUPRIMENTOS DA LOGÍSTICA HUMANITÁRIA 29

3.1. DEFINIÇÃO 29

3.2. Agentes das Cadeias de Suprimentos 32

3.3. MEDIDAS DE DESEMPENHO 35

3.3.1. Medidas de desempenho internas 35

3.3.2. Medidas de Flexibilidade $\quad 35$

3.3.3. Medidas de desempenho externas 36

3.3.4. Custos de estoques 36

3.3.5. Custo de Transporte 36

3.3.6. Distribuição Física 38

4 SISTEMA DE APOIO $\quad 40$

4.1. Características gerais do Modelo Proposto 40

4.1.1. Camada de controle 41

4.1.2. Camada de dados 44

4.1.3. Webservices 50

4.1.4. Camada de Visão 51

4.2. Sistema de ARRECADAÇÃO dE FUndOS 53

4.3. FUnCIONAMENTO dO SISTEMA DE APOIO 55 
5 CONCLUSÃO 


\section{Lista de figuras}

Figura 1 - Totais de Eventos de Desastre de 1974 a $2003 \quad 19$

Figura 2 - Número de pessoas afetadas por desastres naturais $2010 \quad 20$

Figura 3 - Fases do gerenciamento do desastre 20

Figura 4 - Cadeia de Suprimentos na Logística Empresarial 29

Figura 5 - Cadeia de Suprimentos na Logística Humanitária 30

Figura 6 - Agentes da Assistencia Humanitária e Rede de Apoio 33

Figura 7- Arquitetura do Sistema de Apoio 41

Figura 8 - Diagrama de Atividades 44

Figura 9 - Modelo Entidade Relacionamento do Sistema 46

Figura 10 - Modelo Relacional do Sistema 49

Figura 11 - Interface Inicial do Sistema Wordpress 52

Figura 12 - "Plug-in" WP-ecommerce e a conexão pagseguro da UOL 54

Figura 13- Págiana Inicial do Sistema 55

Figura 14 - Página do Sistema após feita a autenticação 56

Figura 15 - Cadastro Usuário 58

Figura 16 - Cadastro Evento $\quad 59$

Figura 17 - Cadastro Fornecedor $\quad 60$

Figura 18 - Cadastro Produto 61

Figura 19 - Cadastro do Local 62

Figura 20 - Consulta dos Produtos Cadastrados 63

Figura 21 - Alocar produto na Cadeia de Suprimentos 63

Figura 22 - Cadastro do Lote 64

Figura 23 - Cadastro do Transporte 65

Figura 24 - Produto para ser alocado na loja virtual 66

Figura 25 - Alocando o produto na loja virual 66

Figura 26 - Loja Virtual do Sistema 67

Figura 27 - Finalização da Compra/doação 67 


\section{Lista de tabelas}

Tabela 1 - Agencias das Organizações das Nações Unidas 26

Tabela 2 - Comparação entre as Logísticas Empresarial e Humanitária 31

Tabela 3 - Características da Logística Humanitária 34

Tabela 4 - Dieta para indivíduo adulto com 2200kcal 43

Tabela 5 - Capacidade útil e custos fixo e variável dos veículos 48 